\title{
Dynamique régressive de la formation forestière de Yamba Berté au Tchad : le crépuscule d'une forêt classée
}

\author{
Frédéric Réounodji ${ }^{\mathrm{a}}$, Man-Na Djangrang ${ }^{\mathrm{b}}, \mathrm{N}^{\prime}$ dilbe Tob-Roc $^{\mathrm{c}}$, Pabamé Sougnabéd
}

RÉSUMÉ. Le maintien et le développement des formations forestières dépendent du degré d'anthropisation et des conditions du milieu. Le Tchad dispose d'importantes formations forestières parsemées dans sa partie sud. Certaines d'entre elles, notamment celle de Yamba Berté, au nord-ouest de la ville de Kélo, ont subi, au cours des dernières décennies, une dynamique régressive préjudiciable à l'équilibre écologique. Cette étude analyse l'évolution de l'occupation du sol et des peuplements ligneux en relation avec les usages et pratiques agropastoraux dans la formation forestière classée de Yamba Berté et autour d'elle. La méthodologie combine une approche analytique des images satellites couvrant la zone d'étude et des enquêtes de terrain sur la perception de la dynamique par les populations. Cette démarche répond au souci de mettre en place un outil diagnostique qui s'appuie sur les données spatiales et sur les perceptions des populations locales pour étudier la dynamique des formations forestières dans une perspective de gestion durable des ressources naturelles en zone de savane. Les premiers résultats montrent que la perception des populations est en conformité avec les caractéristiques et tendances évolutives observées.

ABSTRACT. The maintenance and development of forest formations depend on the degree of anthropization and environmental conditions. Chad has considerable forest formations scattered in the southern part of the country. Some of them, in particular that of Yamba Berté northwest of the city of Kélo, have undergone a regressive expansion over the last decades, that is detrimental to ecological balance. The present study analyzes the evolution of soil occupation and ligneous plantings in relation to agropastoral practices and uses in and around the classified forest formation of Yamba Berté. The methodology combines an analytical approach of the sat ellite images, covering the study area and the field surveys, on the perception of the processes by the populations. Such an approach meets the requirement to set up diagnostic tools using spatial data and to design methods to sustainably manage forest formations in semihumid areas, in line with the people's way of life. The first results show that the perception of populations is in agreement with the evolutionary characteristics and trends observed.

\section{Introduction}

La forêt tropicale couvre environ 1753000 hectares, soit $64 \%$ de la surface totale des forêts dans le monde (Lanly, 1982). Pendant les dernières décennies du $\mathrm{XX}^{\mathrm{e}}$ siècle, le taux de disparition moyen de la forêt dite « naturelle » est estimé à $0,8 \%$ annuellement pour l'ensemble des pays africains (FAO, 1994). Les causes directes de cette situation sont les activités d'aménagement et d'exploitation des ressources forestières.
Antrop (2004) affirme que les forêts se transforment parce que les dynamiques forestières sont l'expression de l'interaction entre des forces naturelles et culturelles de l'environnement.

Par ailleurs, les études réalisées par le programme Écosystèmes et paléoécosystèmes des forêts intertropicales $\left(\mathrm{ECOFIT}^{1}\right)$, ont montré qu'en Afrique centrale, les grandes zones de savane ouvertes au début de l'Holocène ${ }^{2}$ et comblées grâce au retour

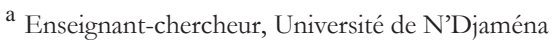

${ }^{\mathrm{b}}$ Enseignant-chercheur, Université de Moundou

c Enseignant-chercheur, Université Adam Barka d'Abéché

${ }^{\mathrm{d}}$ Chercheur, Institut de recherche en élevage pour le développement (IRED) à N’Djaména
} 
de conditions climatiques favorables ont connu des transformations successives correspondant aux différentes étapes de l'utilisation du sol. La modernisation des activités agricoles, traduite par des formes opposées d'intensification ou de déprise, est aujourd'hui la principale cause des mutations qui semblent brutales au regard des évolutions passées.

Cette problématique a fait l'objet de nombreuses études, aussi bien au Cameroun (Achoundong, Youta Happi, Guillet, Bonvallot et Kamgang Belaya, 2000; Aoudou Doua et Tchotsoua, 2010; Tchotsoua, 1999; Youta Happi, 1998; Ntoupkap, 1994), en Côte d'Ivoire (Yao Sadaiou Sabas et collab., 2009), au Gabon (Fredou et Maley, 2000) ou en République démocratique du Congo (Schwartz et collab., 1996). D'emblée, l'hypothèse selon laquelle l'action humaine est importante sur la dégradation des ressources forestières est apparue plausible. Sans donner un trop grand crédit aux théories liant la déforestation observée à la seule action de l'humain (besoin de combustible, feux de brousse utilisés comme technique de chasse, etc.), il n'en demeure pas moins qu'on peut désormais indexer les périodes sèches qui conduisent la plupart du temps à l'apparition de la savane (formation végétale composée surtout d'herbes) et les conditions climatiques favorables entrainant la reprise forestière (Téblé, 2014; Seignobos, 1980). L'idée d'un équilibre arrêté et définitif relève de la fiction; la dynamique régressive semble dominante (Djangrang et Retaillé, 2015; Raimond, Robert et Garine, 2012; Andigué, 1999; Boutrais, 1980; Marchal, 1980).

Malgré la tendance à la reprise des formations forestières par endroits, mise en évidence par différentes méthodes (comparaison de photographies aériennes ou d'images satellites), de larges étendues de savane dominées par la végétation arbustive et/ou herbeuse persistent sous forme de mosaïques à différents endroits du Tchad. Dès les années 1950, l'administration coloniale a pu en identifier plus d'une dizaine, dont la forêt de Yamba Berté (Cabot, 1965).

Située au sud du département de la Kabia (province de Mayo-Kébbi Est) et à cheval entre les départements de Mayo Dallah (province de Mayo-Kébbi Ouest) et de la Tandjilé Ouest (province de la Tandjilé), la forêt de Yamba Berté est une forêt classée selon l'arrêté $n^{\circ} 30 / \mathrm{SF}$ du 15 janvier 1955 portant classement de la forêt de Yamba Berté. Elle s'étend sur six cantons (à savoir Erdé, Tagal, Berem, Kordo, Gagal et Mesmé) et couvre une superficie d'environ 64000 hectares (voir figure 1). Cette formation forestière était autrefois dominée par différentes espèces ligneuses telles que le bouleau d'Afrique (Anogeissus leiocarpus) et le marula (Sclerocarya birrea), associées aux Combretum glutinosum sp., caillcédrat (Khaya senegalensis), kapokier (Bombax costatum) et acacia (Faidherbia albida), spécimens types du domaine sahélo-soudanien et, dans une moindre mesure, au prunier d'Afrique (Sclerocarya birrea) en peuplements presque purs. Présentement, cette formation forestière fait place à un ensemble végétal complètement dégradé et épars. Dans ce contexte, il importe de s'interroger sur les facteurs à l'origine de la disparition de ces ressources ligneuses.

L'objectif de cette étude est de faire un état des lieux de la forêt classée de Yamba Berté et de caractériser son évolution sous l'effet des activités anthropiques à l'aide de la télédétection. 


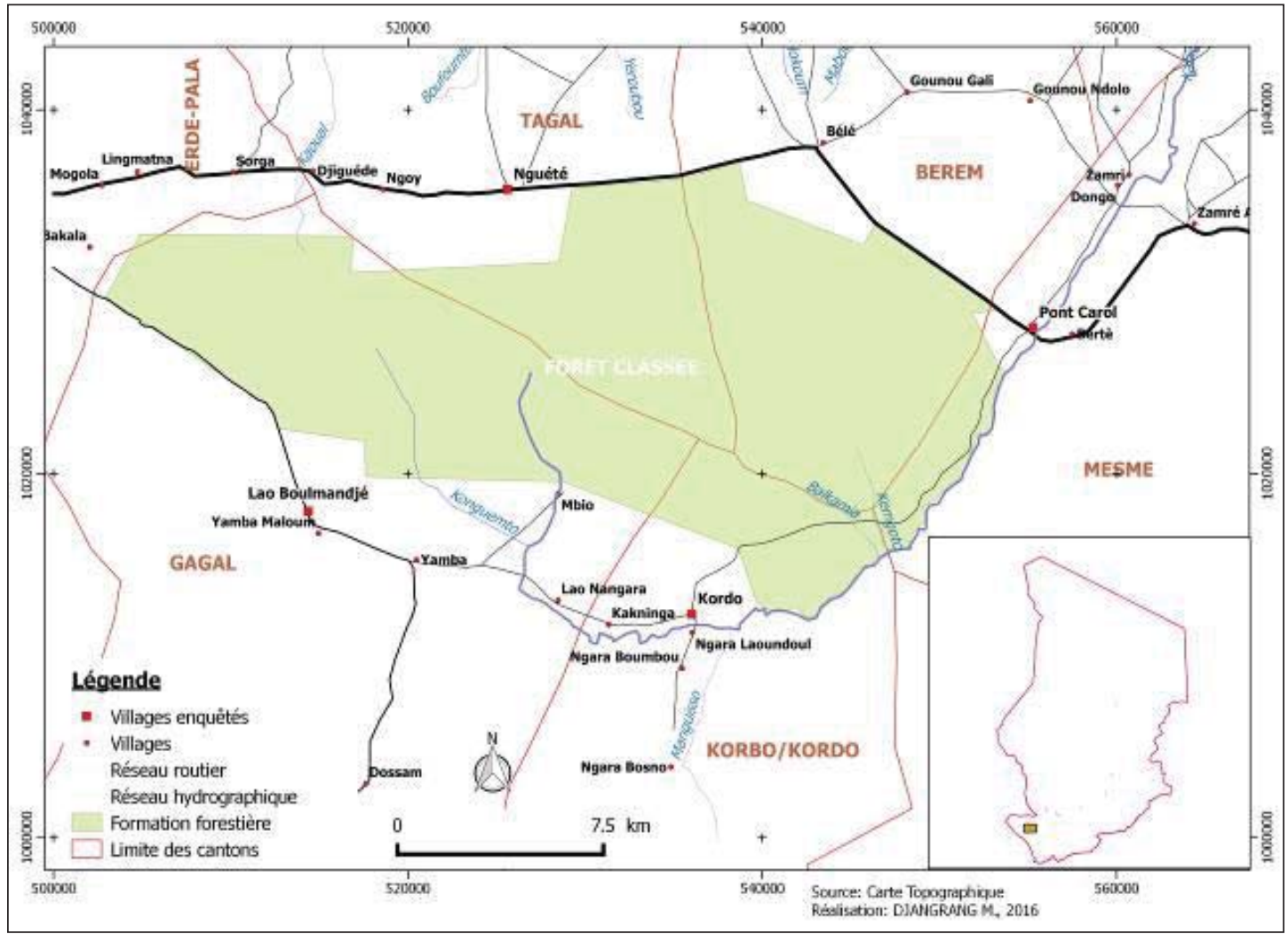

Figure 1 - Localisation de la forêt classée de Yamba Berté Source : Djangrang, 2016

\section{Matériel et méthodes}

Quatre sites d'étude ont été choisis : Pont Carol, Lao Boulmandjé, Kordo et Nguété (voir figure 1). Ils sont représentatifs de la diversité de la formation forestière et des gradients de l'utilisation du sol. Des enquêtes sur les pratiques agropastorales - pratiques associant les activités agricoles et d'élevage sur le même espace - ont été réalisées auprès de 50 chefs de ménage répartis sur les quatre sites.

Les questions sont orientées sur la perception, par les populations, de l'évolution de la végétation ligneuse, des pratiques agropastorales et de leurs possibles interactions sur la disparition des espèces ligneuses de la forêt de Yamba Berté. L'analyse est étroitement dépendante du résultat de l'analyse de deux images satellites tirées du programme Landsat ${ }^{3}$ et acquises par le groupe de recherche du Laboratoire de cartographie et d'études géographiques de l'Université de Moundou, respectivement en 2000 et 2013. Lors du traitement, nous avons appliqué une classification dite « supervisée » sur les deux images Landsat.

Revue Organisations \& Territoires • Volume $28 \cdot$ No $_{2} \cdot 2019$
Notre nomenclature distingue deux grands types de milieux (sols et végétation) inspirés de la division en grands thèmes du programme Corine Land Cover ${ }^{4}$ (CEC, 1993) et de l'éclatement modulo-hiérarchique du projet Africover $(\mathrm{FAO}, 1997)$ : territoires agricoles (jachère et champ), forêts et milieux semi-naturels (sol nu, savane arborée et savane arbustive). Lorsque cela s'est avéré nécessaire et réalisable, notamment pour les territoires agricoles et les forêts, nous nous sommes attachés à préciser l'occupation des sols au sein de ces deux types lors de l'analyse des images satellites de 2000 et 2013. L'exactitude et la fiabilité de nos résultats ont été évaluées à partir d'une matrice de confusion, estimée respectivement entre 0,7476 et 0,7136 .

\section{D'un paysage de savane boisée à un paysage fortement anthropisé}

Les figures 2 et 3 présentent les cartes d'occupation du sol, obtenues après l'analyse des images satellites Landsat acquises en 2000 et 2013. Ces figures montrent l'évolution temporelle des surfaces correspondant à cinq types d'occupation du sol identifiés, à savoir le sol nu (12,47 \% en 2000 et $24,22 \%$ en 2013), 
la savane arborée ( $42 \%$ et $29,55 \%$ ), la savane arbustive $(21,43 \%$ et $21,65 \%)$, les jachères $(14,53 \%$ et $13,05 \%)$ et les champs $(9,57 \%$ et $11,54 \%)$ ainsi que la proportion du paysage occupée par les différentes catégories d'unités d'occupation du sol (voir tableau
1). Les deux figures montrent également les relations qui existent entre la surface totale des cinq types d'occupation du sol et les taches correspondantes. Les résultats soulignent des différences importantes dans l'état de la structure de l'occupation du sol.

\begin{tabular}{|l|c|c|c|c|c|c|}
\hline \multirow{2}{*}{$\begin{array}{l}\text { Types d'occupation } \\
\text { du sol }\end{array}$} & \multicolumn{3}{|c|}{$\mathbf{2 0 0 0}$} & \multicolumn{3}{c|}{2013} \\
\cline { 2 - 7 } & $\begin{array}{c}\text { Surface } \\
\text { (ha) }\end{array}$ & $\begin{array}{c}\text { Nbre de par- } \\
\text { celles }\end{array}$ & $\begin{array}{c}\text { \% } \\
\text { Sol nu }\end{array}$ & $\begin{array}{c}\text { Surface } \\
\text { (ha) }\end{array}$ & $\begin{array}{c}\text { Nbre de par- } \\
\text { celles }\end{array}$ & \% \\
\hline Savane arborée & 28576 & 1506 & 12,47 & 16457 & 1873 & 24,22 \\
\hline Savane arbustive & 14560 & 1903 & 21,43 & 14710 & 1965 & 21,65 \\
\hline Jachères & 9876 & 1218 & 14,53 & 8867 & 2822 & 13,05 \\
\hline Champs & 6504 & 833 & 9,57 & 7842 & 1458 & 11,54 \\
\hline Total & 67956 & & 100,00 & 67956 & & 100,00 \\
\hline
\end{tabular}

Tableau 1 - État de l'occupation du sol en 2000 et 2013

Source : Images satellites Landsat de 2000 et 2013

En 2000 (voir figure 2), le paysage apparait plutôt rural, dominé par des espaces couverts de savane boisée $(63,43 \%)$ et par les jachères $(14,53 \%)$. La relation surface/nombre de parcelles révèle une grande quantité de savanes (3 363 ha), distribuées sur une surface de 4310 hectares, en opposition à une petite superficie herbacée (8 476 ha) contenant un nombre réduit de parcelles (1 506). Cela indique la faible présence d'une activité agricole (8 476 ha) sur une surface parcellaire peu morcelée (833) à l'est et le long de la route principale entre Kélo et Pala. Quelques incursions sont aussi observées à l'intérieur de la formation forestière à partir des villages de Nguété, Ngoy et Sorga.
En règle générale, cultures et jachères se distribuent de façon concentrique et compacte. Elles constituent des associations sur de grandes surfaces, seulement interrompues par des habitats et des chemins. Les surfaces arborées occupent 2854 hectares et sont disposées en bandes allongées dans le sens est-ouest, lesquelles se fragmentent vers les deux extrémités. Vers le centre-est, et sur une aire de 1456 hectares, les parcelles de savane arbustive s'alignent le long de la savane arborée; elles sont fréquemment interrompues par des champs. Les champs, localisés en bordure des zones arbustives, représentent $9,57 \%$ de l'ensemble paysager.

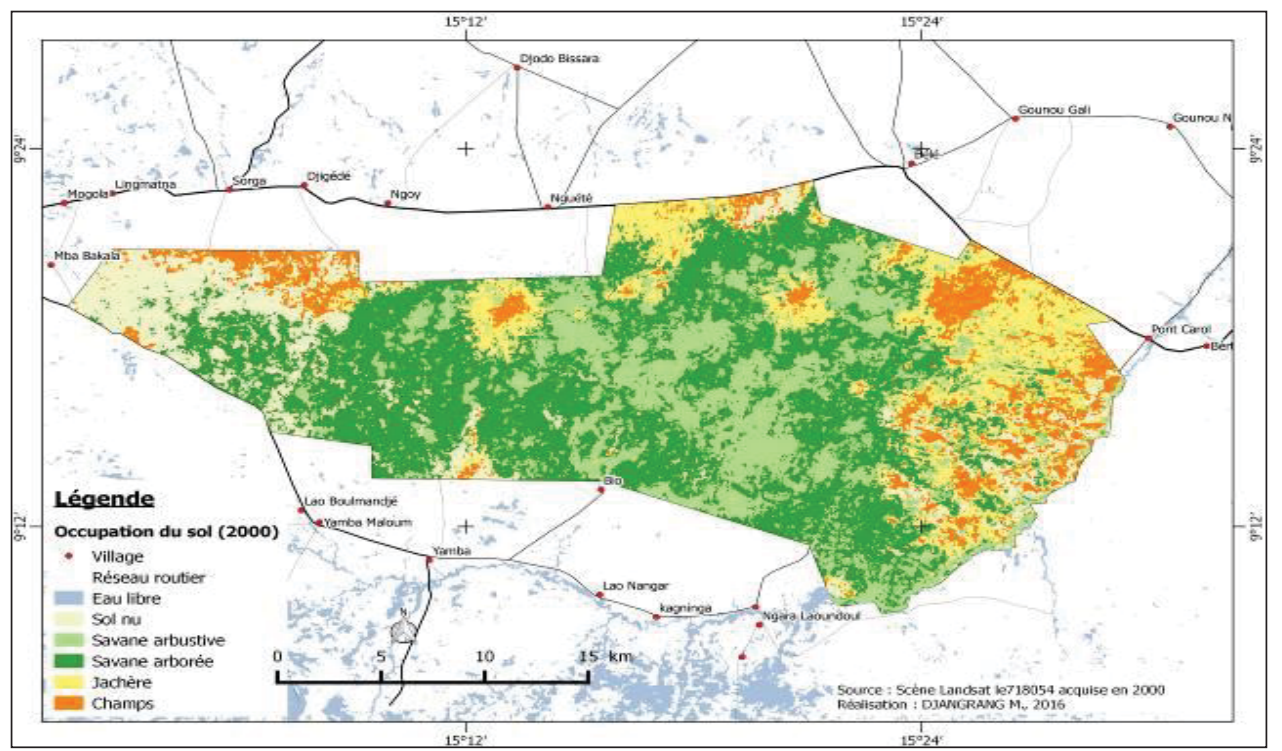

Figure 2 - Occupation du sol en 2000 Source : Djangrang, 2016 
En 2013 (voir figure 3), le paysage a subi d'importantes transformations, traduites principalement par le recul et par la fragmentation de la savane arborée. Le nombre de parcelles et l'aire de cultures ont augmenté de 833 à 1458 parcelles et de 6504 à 7842 hectares $(11,54 \%)$. Dans le cas de la végétation herbacée, représentée par le type sol nu, on assiste aussi à un dédoublement de sa surface, soit de 8476 à 16457 hectares (24,22\%), ainsi qu'à une augmentation de la taille des parcelles herbacées liée à l'accroissement de leur nombre (1 506 à $1876)$.

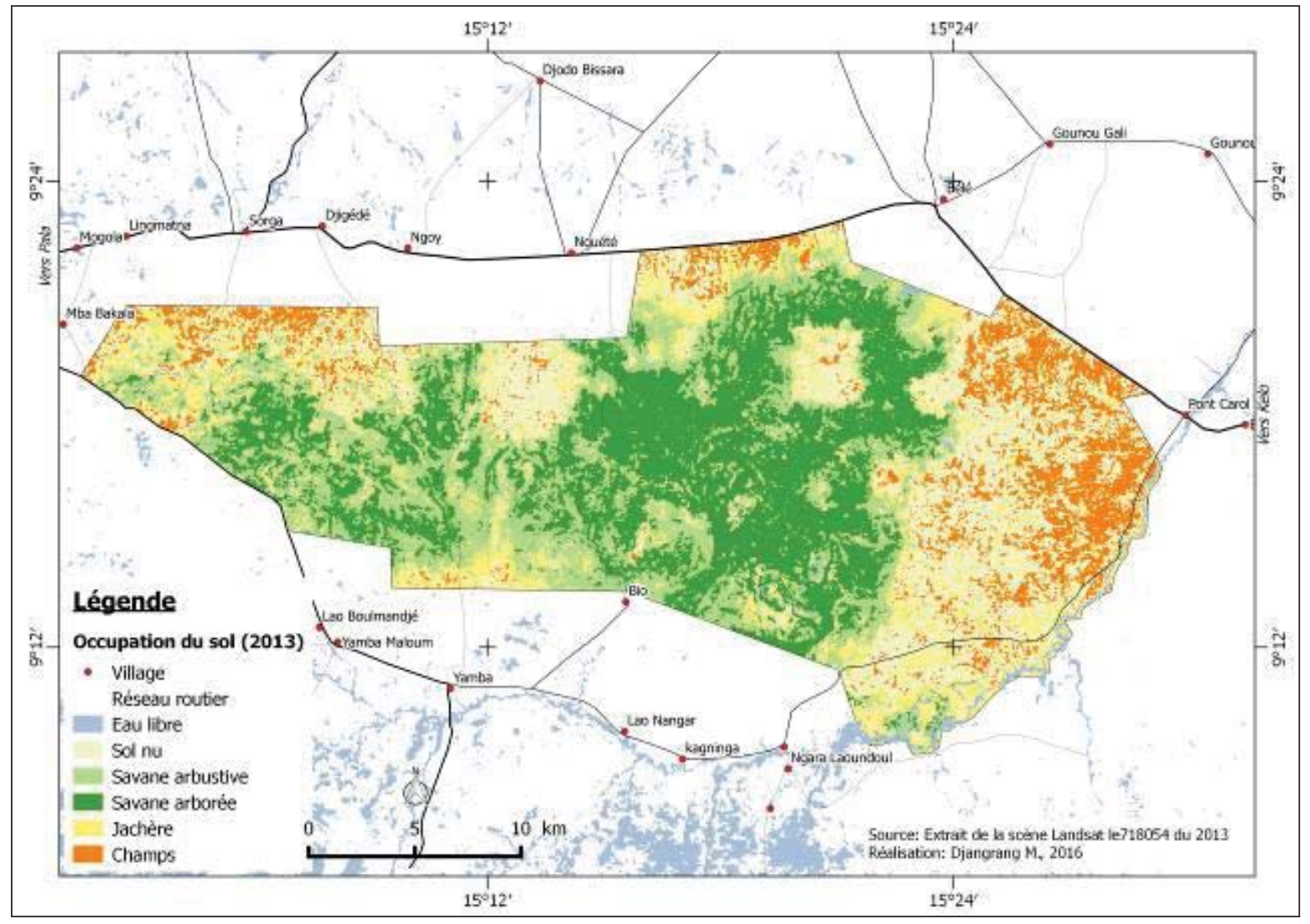

Figure 3 - Occupation du sol en 2013 Source : Djangrang, 2016

Les raisons de cette évolution spectaculaire sont liées à la stratégie de développement imposée par les effets de la croissance démographique. Les deux cartes d'occupation du sol montrent que, depuis 2000, on a intensément exploité la terre à des fins agricoles et de grandes surfaces ont été défrichées. Les boisés de l'intérieur ont été moins affectés qu'aux alentours des villages. La faible densité de chantiers forestiers et l'augmentation du prix du bois ont généré une très forte pression sur les ressources ligneuses les plus accessibles de la formation forestière. L'intensification du déboisement a généré une augmentation de l'érosion des sols.

\section{Facteurs de la dynamique régressive de la forêt classée}

Plusieurs facteurs sont responsables de la dynamique régressive de la forêt classée de Yamba Berté, dont l'expansion de l'élevage pastoral et l'ouverture d'un front pionnier dans la zone, en raison de l'immigration des populations venues des bassins cotonniers surexploités des pays marba, moussey et ngambaye.

\section{1 Évolution des pratiques d'élevage et accroissement du cheptel}

Jusqu'aux années 1970, la forêt classée de Yamba Berté était située bien au-delà de la limite sud de l'élevage pastoral ${ }^{5}$. En effet, les pasteurs ne voulaient pas 
courir le risque d'y effectuer la transhumance en raison de son statut de forêt classée et, surtout, de la présence de la trypanosomiase (maladie animale et humaine transmise par un vecteur appelé mouche tsé-tsé ou glossine) qui sévissait dans cette zone humide. Avec la sécheresse des années 1970, la glossine se faisant moins menaçante, les pasteurs des peuples djafoun ${ }^{6}$ et wodaabé ${ }^{7}$ du Niger et ceux du Nigeria ont commencé à investir les environs de la forêt classée. Aujourd'hui, trois systèmes d'élevage cohabitent dans la région : le système transhumant, le système agropastoral et le système agricole.

\subsubsection{Le système transhumant}

La transhumance est un système d'élevage basé sur la mobilité des troupeaux en réponse aux conditions climatiques difficiles des milieux semi-arides et à la dispersion spatio-temporelle des ressources pastorales. Elle s'effectue généralement entre un terroir " d'attache ${ }^{8}$ » (en saison des pluies) et des terroirs « d'accueil ${ }^{9}$ » en saison sèche, sur des distances qui peuvent aller de quelques dizaines à plusieurs centaines de kilomètres (Sougnabé, 2010). Ce système d'élevage est présent dans la forêt classée de Yamba Berté depuis les décennies 1980 et 1990.

Les transhumants sont des pasteurs arabes venus de Dourbali et de Melfi, dans le centre du pays, et auxquels s'ajoutent les Foulata ${ }^{10}$ de la province du Chari-Baguirmi et les M'bororo du Nigeria. Chaque année, lors de leurs déplacements, ces éleveurs marquent toujours un arrêt dans cette forêt avant de poursuivre leur périple à Béinamar ou à Gagal (Tchad). Ils dépassent parfois la frontière nationale pour rejoindre le Cameroun ou la République centrafricaine. À partir de juin, ils remontent vers le nord en direction des villes de Fianga et Gounou Gaya, ou plus au sud vers Gagal, Goumadji et Beinamar au Tchad (voir figure 4).

Ce système d'élevage est pratiqué généralement par des pasteurs disposant de grands troupeaux de cheptel constitués surtout de gros ruminants. C'est un système d'élevage très redouté des agriculteurs sédentaires en raison des dégâts importants que les animaux causent aux cultures (Réounodji, 2003). En dépit des risques d'amendes et d'emprisonnement qu'ils encourent, ces éleveurs bravent l'interdiction et envahissent la forêt à la recherche de pâturages verts. Certains se sont même vu confier des animaux appartenant aux chefs militaires résidant en ville.
La pression régulière qu'exercent les troupeaux de bovins sur les ressources forestières finit par dégrader peu à peu la réserve de Yamba Berté.

\subsubsection{Le système agropastoral}

Le système agropastoral combine l'agriculture à l'élevage et connaît une forte croissance en raison de la poussée démographique et de la sédentarisation progressive des éleveurs mobiles dans cette partie du pays. Il se distingue du système transhumant par un habitat fixe dans le terroir d'attache, où réside une partie de la famille, alors que certains de ses membres effectuent la transhumance saisonnière.

Dans ce système, on retrouve les Foulata, qui sont installés dans des féricks (groupements de nomades) permanents plus proches de la réserve forestière de Yamba Berté, en particulier dans les villages de Pont Carol, Kordo, Yamba Malloum, Goula, Sorga, Ndjigette, Ngoye, Ngetté I et Belé Vanza. Il y a aussi les Silsilbé (parlant le haoussa) dans le canton de Koumou. Ces deux groupes d'éleveurs partagent le même espace pastoral en saison des pluies de même qu'en saison sèche. Il en résulte une augmentation significative du nombre d'animaux dans les alentours immédiats de la réserve forestière. Du coup, la pression pastorale issue des deux systèmes d'élevage sur les formations forestières de la réserve est importante.

\subsubsection{Le système agricole}

Le système agricole est une caractéristique connue de la région, où des agriculteurs capitalisent dans le cheptel afin de se prémunir contre les périodes plus difficiles. Ces agriculteurs élèvent quelques têtes d'animaux comme source de revenus d'appoint. Le bétail s'inscrit dans une économie qui correspond souvent à un degré d'enrichissement du producteur agricole.

Ce mode d'élevage, qui gagne de plus en plus de terrain, semble avoir un impact évident sur les ressources ligneuses de la forêt de Yamba Berté. En saison sèche, les animaux sont laissés libres dans la forêt et les champs pour qu'ils consomment les feuilles et les résidus des récoltes. En saison des pluies, les bœufs sont envoyés dans les pâturages éloignés des auréoles de cultures, plus en profondeur de la forêt classée de Yamba Berté. 


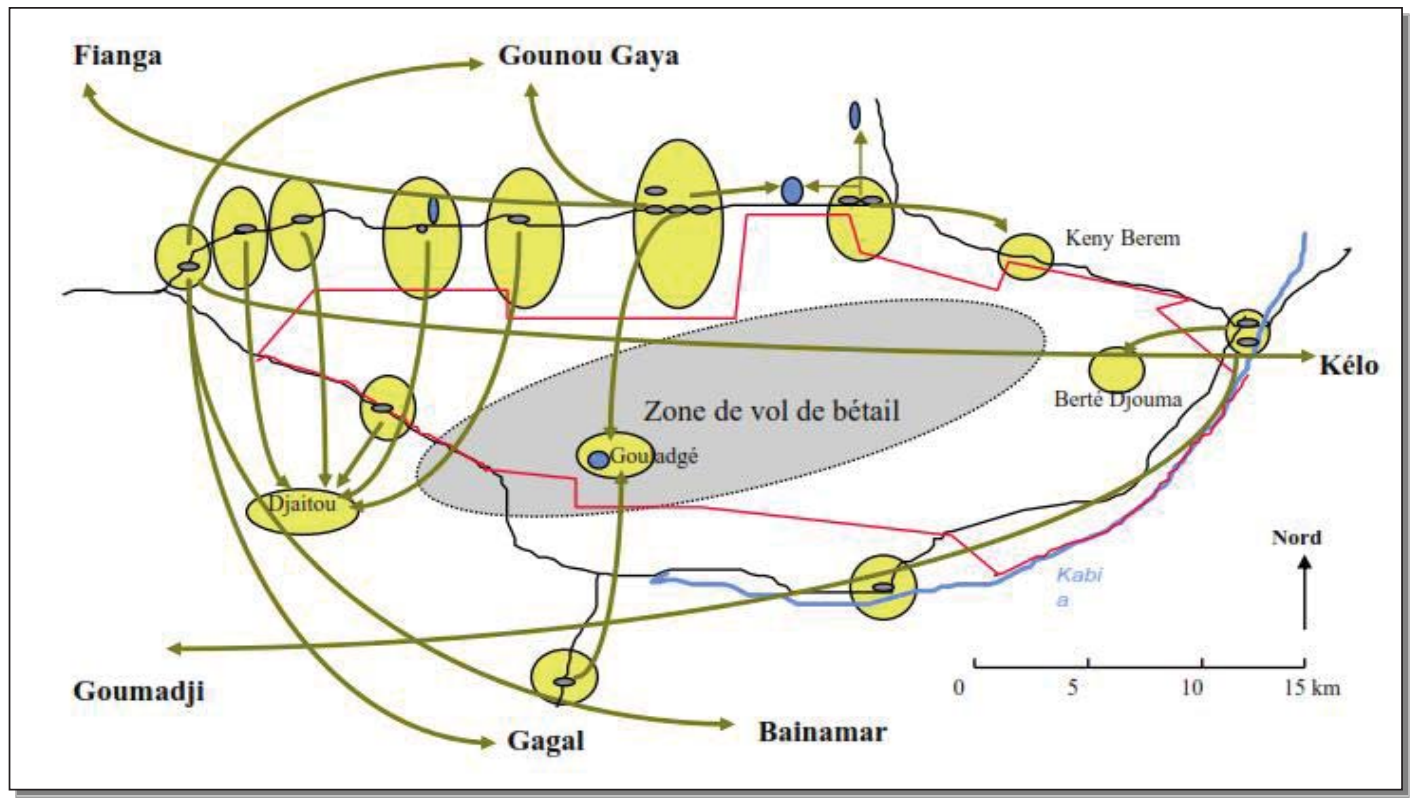

Figure 4-Zones de pâturage en saison sèche dans la forêt de Yamba Berté Source : Sougnabé, Ali Brahim et Hassane Mahamat, 2004

En 2012, le survol aérien a dénombré 48 troupeaux comptant jusqu'à 5904 bovins dans la forêt de Yamba Berté et ses environs immédiats. Au cours de la même période, les statistiques de la Direction agricole du ministère de l'Agriculture donnent un effectif de 6800 têtes de bovins (Sougnabé et collab., 2004). En 2016, on dénombre dans le secteur nord de la forêt classée au-delà de 56 troupeaux comprenant un effectif de 6888 têtes de bovins, d'octobre à décembre, ainsi que 64 troupeaux comprenant un effectif de 7872 têtes de bovins de mars à mai. Sur l'ensemble de la forêt classée et ses alentours, l'effectif du cheptel bovin peut atteindre les 13000 têtes, soit 10400 unités de bétail tropical (UBT).

L'unité de bétail tropical a pour critère de base une tête de bétail d'un poids de $250 \mathrm{~kg}$ consommant $6,5 \mathrm{~kg}$ de matière sèche par jour. Sur cette base, au Tchad, l'UBT officiellement admise est établie pour le bovin à 0,8 ; c'est-à-dire que la valeur d'un bovin est égale à 0,8 UBT $(1$ bovin $=0,8$ UBT). Cette valeur varie d'un pays à un autre.

La capacité de charge d'un pâturage est une notion indispensable à prendre en considération pour évaluer le niveau de pression sur les ressources forestières. Ainsi, la capacité de charge pour la forêt classée, calculée à partir des données fournies, est de 1,60 UBT/ha par année de pâture. Elle est donc très élevée. Il en résulte une forte pression exercée par le bétail sur les ressources forestières, quand on sait que des espèces ligneuses telles que le doussié (Afzelia africana) et le cailcédrat sont sauvagement élaguées pour nourrir le bétail.

La question de l'élevage dans la forêt classée de Yamba Berté et autour d'elle se pose aujourd'hui en regard de l'accès aux ressources pastorales, en dépit de la présence de brigades de répression mises sur pied dès sa création par les services des eaux et forêts. La pression animale croissante, liée à l'augmentation du cheptel sédentaire et à l'afflux d'animaux transhumants, constitue un véritable obstacle à l'effort de conservation des ressources forestières de cette réserve nationale, qui est aujourd'hui au crépuscule de son existence.

\subsection{Ouverture d'un front pionnier agricole dans la zone}

\subsubsection{Les marges forestières investies par des migrants}

L'afflux des migrants depuis les régions densément peuplées, où la potentialité de production agricole a été mise à mal par la pression anthropique, a abouti à l'installation d'un nouveau front pionnier plus proche de la forêt classée de Yamba Berté. Ces mouvements des populations ont été amplifiés par la sécheresse du milieu des années 1980, lesquels mouvements ont 
conduit à la colonisation des marges de cette formation forestière par des familles venues des pays marba, moussey et zimé. Attirées par la potentialité agricole offerte par des terres fertiles, ces familles d'agriculteurs se sont mises à défricher des hectares autour de la forêt pour leurs cultures. Les marges de la forêt sont ainsi prises d'assaut; la réserve elle-même est désormais menacée par l'agriculture.

À la fin des années 1980, de nouveaux villages se créent à la suite de l'immigration des peuples marba, ngambaye, zimé et moussey. Les densités deviennent fortes: les terres sont cultivées de façon permanente et sont même partagées entre ces ethnies (voir figure 5). À la longue, la pression sur le foncier agricole est tellement forte que les terres cultivées ne sont plus mises en jachère, même au-delà de cinq années de culture.
Jusqu’à la fin des années 2000, le Programme de conservation et de gestion des ressources naturelles (PCGRN), financé par la Deutsche Gesellschaft für Technische Zusammenarbeit (GTZ), a recensé environ 57 villages riverains de la réserve, alors que, pendant les années 1960, la périphérie de la forêt classée était inhabitée, depuis Pont-Carol jusqu'à Gagal, dans le département de la Haute Kabia (Cabot, 1965). Entre 1993 et 2003, les densités autour de la formation forestière, où se trouve la plus forte concentration humaine, dépassent les $170 \mathrm{hab} . / \mathrm{km}^{2}$. Par comparaison aux cantons des peuples marba, ngambaye et moussey, les densités humaines varient de 50 à 70 hab. $/ \mathrm{km}^{2}$ (Sougnabé et collab., 2004). Il en résulte que ces nouvelles densités humaines augmentent inévitablement la pression sur les ressources naturelles, ce qui conduit à la déforestation et à la dégradation de l'écosystème forestier de Yamba Berté.

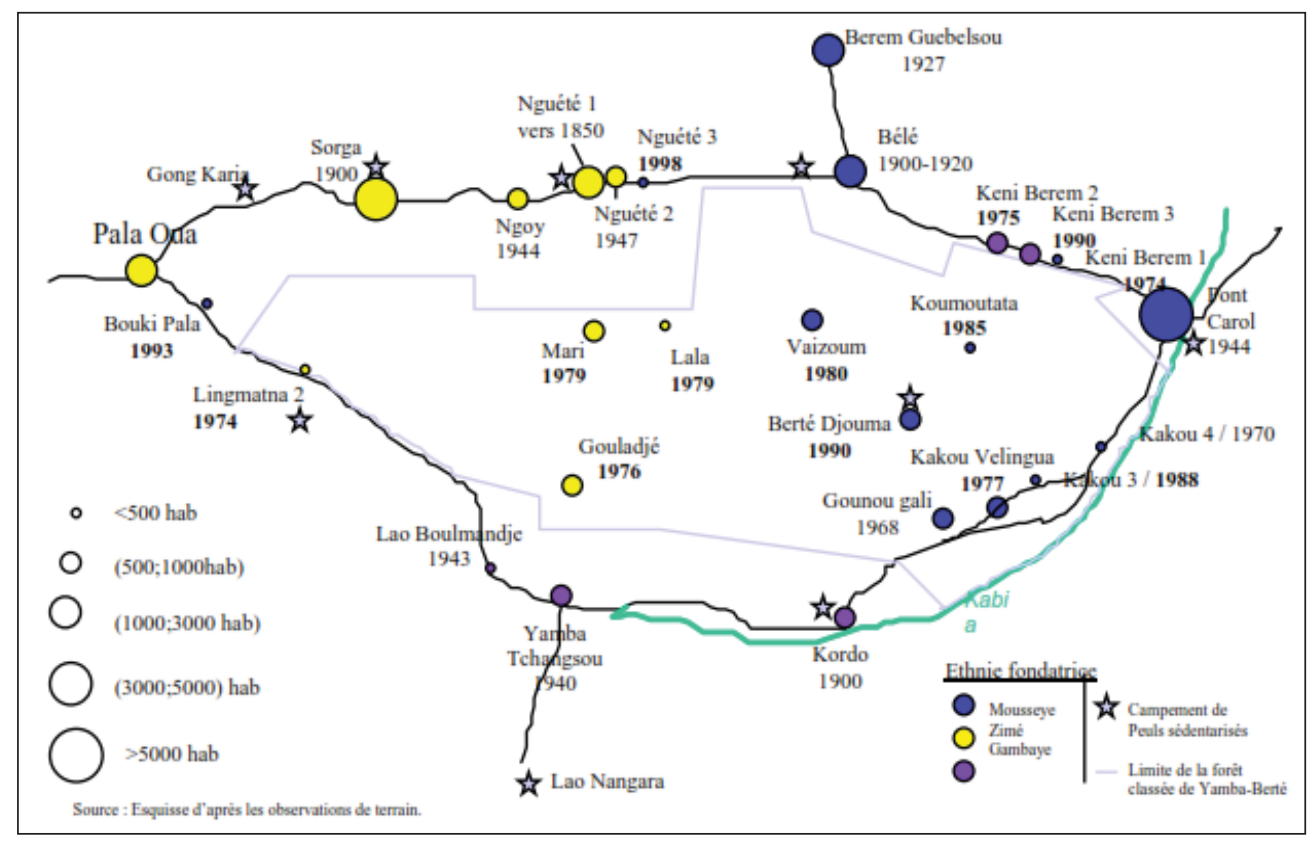

Figure 5- Répartition des villages et des campements peuls Source : Sougnabé et collab., 2004

\subsubsection{L'évaluation de la dynamique forestière par transect}

À Pont Carol, situé à l'est, à environ $1 \mathrm{~km}$ de la formation forestière de Yamba Berté, le transect (diagramme en profil, c'est-à-dire la ligne virtuelle qui montre le profil du terrain) est dominé par la strate herbeuse, avec une densité moyenne de 31 individus d'arbustes par placette ${ }^{11}$ et un recouvrement moyen de $17,36 \%$ du territoire. Les espèces d'arbres caractéristiques de ce transect sont Isoberlinia doka, Burkea africana et le bouleau d'Afrique (Anogeissus leiocarpus). L'exploitation du faciès ${ }^{12}$ dans lequel est implanté le transect de Pont Carol pour l'agriculture, le pâturage de saison sèche et la coupe de bois serait la principale cause de la baisse du taux de recouvrement de la strate boisée. Par contre, le nombre élevé d'individus de la strate arbustive provient des rejets d'arbres coupés pour des besoins domestiques. 
Dans la zone de Nguété, située au nord de Pont Carol, la végétation où est situé le transect est caractérisée en général par une strate arbustive dont le taux de recouvrement moyen est de $10 \%$ et dont la densité moyenne est de 35 individus par placette. Plus spécifiquement, la placette implantée à Nguété présente un taux de recouvrement moyen de 6,08\% pour la strate arbustive, de 3,78 \% pour la strate arborée et de $0 \%$ pour la strate boisée. Les espèces caractéristiques du transect sont : le kinkéliba (Combretum sp.), Daniella oliveri, le cailcédrat et le karité (Butyrospermum parkii).

Un peu plus loin, à $2 \mathrm{~km}$ du village de Nguété, sur un sol de koro (sol ferrugineux latéritique), la strate arbustive impose une grande représentativité de ce secteur écologique, avec 243 individus et un taux de recouvrement de 61,5\%. La strate arborée, quant à elle, a un taux de recouvrement de $27 \%$. La strate boisée est la plus faible, soit $12 \%$ du territoire, pour la simple raison que les grands arbres sont détruits lors des défrichements agricoles.

À environ $6 \mathrm{~km}$ du village de Lao Boulmandjé, sur des sols sablo-argileux et limoneux-argileux, les espèces caractéristiques sont le kinkéliba, Terminalia macroptera ainsi que le Ficus sp. Les buttes les plus larges et les plus élevées gardent parfois des variétés plus typiquement soudaniennes, par exemple le cailcédrat, le kapokier et le karité. Le milieu est fortement marqué par les activités agropastorales. La végétation ligneuse est dominée par un faciès de savane arbustive faiblement dense. La strate arbustive domine à $96 \%$ le transect, tandis que la strate boisée est quasi inexistante.

L'absence de la strate boisée et la faible représentation de la strate arborée sont liées à la proximité du centre urbain. La présence des sols limono-sableux et argilo-sableux ainsi que d'une bioturbation importante, marquée par la présence des turricules de vers de terre qui à̀rent le sol, expliquerait le pourcentage de la strate arbustive. Toutefois, la forte pression de l'activité agropastorale et du feu de brousse a contribué au maintien et à l'expansion des surfaces dénudées, qui sont passées de 8476 à 16457 hectares, soit une régression de plus 846 hectares de savane arbustive (voir figure 3).

Sur l'ensemble de la zone couverte par la formation forestière de Yamba Berté, la végétation est aujourd'hui une savane arbustive fortement dégradée. La strate boisée est très faiblement représentée, soit
1471 hectares en 2013, alors qu'elle était de 1456 hectares en 2000 (voir figure 2). Cette faible couverture de la végétation boisée s'explique par une forte pression humaine observée actuellement sur la formation forestière.

En 2002, les zones agricoles (zones de culture et jachères) et les habitations représentaient environ $57 \%$ de la superficie totale de la forêt classée de Yamba Berté. Les formations forestières sont estimées en 2018 à $43 \%$ et la faune terrestre serait en disparition. Les facteurs de dégradation indexés sont le défrichement des terres de cultures, le pâturage et les feux de brousse. À cela s'ajoute l'exploitation incontrôlée du bois et du charbon de bois, en particulier dans les secteurs de Pont Carol et de Bélé.

De manière générale, la pression anthropique a eu des répercussions négatives sur les facteurs de production, occasionnant une pénurie des terres cultivables, une réduction du temps de jachère et une baisse de la fertilité des sols. Du coup, les productions agricoles ont considérablement chuté. Partout dans la région, les rendements sont habituellement de l'ordre de $460 \mathrm{~kg} / \mathrm{ha}$ pour le sorgho et de $350 \mathrm{~kg} / \mathrm{ha}$ pour le mil, tandis que la production moyenne de ces céréales est de $200 \mathrm{~kg}$. Ce faible niveau de production est loin de couvrir les besoins alimentaires des familles.

\subsubsection{Le développement de la culture du coton}

Depuis son indépendance en 1960, le Tchad a voulu faire du secteur rural le pilier du développement économique en y consacrant une large part de son budget. Pour les pouvoirs publics, seule une production soutenue des cultures de rente permettrait au pays de se hisser sur le marché international, puis de générer suffisamment de revenus pour faire face aux enjeux du développement. C'est pourquoi le principal objectif visé par les projets de développement agricole est d'accroître la productivité agricole.

Du coup, la région entourant la forêt classée de Yamba Berté a été le principal bastion des cultures d'exportation, en particulier le coton. Les cantons de Kordo, Mesmé, Gagal, Erdé-Pala et Tagal, habités des peuples parlant le mesmé, le moussey, le djimé, le pévé, le doué, le dari et le kado, sont considérés depuis des décennies parmi les grands bassins producteurs de coton-graine au Tchad. Ces 
entités territoriales ont été identifiées par les autorités parce qu'elles incarnaient un pouvoir fort et durable. Ce pouvoir a permis aux chefs de canton d'assurer la sécurité foncière et l'accroissement des productions agricoles par les sociétés paysannes, ce qui a rendu possibles de fortes productivités vers les années 1950.

La région a été la principale pourvoyeuse en cotongraine de la société Coton'Tchad, avec une production moyenne de plus de 420 tonnes durant les années 1970 (Cabot, 1965) et de 370 tonnes dans les années 1980. Bien que la production cotonnière ait périclité à partir des années 1990 en raison de la conjoncture économique, il n'en demeure pas moins que cette culture a imprimé sa marque dans le paysage agraire à travers la destruction du couvert végétal, dont elle est la principale responsable.

En favorisant les défrichements de la savane pour accroître les superficies cultivées, les paysans ont délibérément empiété sur la limite de la forêt classée de Yamba Berté. Cette dynamique de modernisation agricole pose de manière claire le problème de dégradation inquiétante des ressources forestières.

\section{Conclusion}

L'analyse diachronique des images satellites Landsat datées de 2000 et 2013 a permis de mettre en évidence la dynamique régressive de la forêt classée de Yamba Berté. Cette formation forestière, qui était estimée à plus de $95 \%$ de la superficie totale de la réserve à la fin des années 1950, s'est considérablement dégradée, à telle enseigne que la végétation arborée ne représentait que $42 \%$ de la superficie totale en 2000. En 2013, elle ne constituait plus que $29 \%$ de la superficie.

D’année en année, la superficie de la forêt classée diminue sous l'emprise des activités agricoles et pastorales. En effet, les superficies cultivées ont également progressé entre 2000 et 2013 en raison de l'augmentation des densités démographiques alimentées par l'immigration et de la charge pastorale observée durant la même période (en tout, 7981 hectares ont été déboisés en moins de 13 ans, soit $613,9 /$ ha par an).
Toute cette dynamique finit, comme nous l'avons déjà mentionné, par exercer une forte pression sur les ressources forestières. La dégradation de la couverture végétale est bien visible dans le paysage à travers l'apparition des sols nus et la prédominance d'arbustes qui colonisent les jachères. Les formations forestières naturelles ont cédé progressivement leur place aux formations anthropisées, c'està-dire des formations naturelles modifiées par les activités humaines. Dans l'ensemble, l'écologie de la formation forestière de Yamba Berté est très fragilisée. Sur des butes exondées, les sols sont presque dénudés, laissant apparaitre par endroits des cuirasses ferrugineuses, pauvres et très sensibles au lessivage et à l'érosion hydrique. Les plaines sont progressivement colonisées par les espèces arbustives. De nombreux espaces boisés sont exposés à l'érosion hydrique.

Outre la sécheresse consécutive aux aléas climatiques, l'agriculture induite par l'essor démographique et le surpâturage (abattage et dessouchage, broutage, piétinement et émondage excessifs) constituent les principaux facteurs de dégradation de la forêt classée de Yamba Berté.

Notre étude a démontré et prouvé la dynamique régressive de cette forêt, dont la tendance se poursuit de manière inquiétante. Cette situation est d'autant plus préoccupante que les efforts menés par les pouvoirs publics chargés de la protection de l'environnement et des projets de développement n'ont pas permis de freiner de manière durable la pression anthropique sur ce massif forestier, actuellemet au crépuscule de son existence. Si cette tendance se maintient, la forêt classée de Yamba Berté risque de disparaitre pour céder la place aux activités humaines, à l'instar de la réserve de faune de Mandelia ou de la réserve de chasse de Douguia dans les environs de N'Djamena.

La réhabilitation de la forêt de Yamba Berté passe par la matérialisation de sa limite, comme préalable à la relocalisation des populations et à la mise en valeur adéquate des terres. Une telle action nécessite la forte implication et la responsabilisation des populations locales et de tous les usagers des ressources naturelles dans la gestion concertée de cette forêt, dont la survie est profitable à tous les acteurs. 


\section{NOTES}

1 ECOFIT est un programme de la FAO qui étudie l'évolution des forêts tropicales sur de longues durées. Il aborde le problème aux échelles de temps de la période chaude interglaciaire qui a débuté il y a 10000 ans (Holocène). ECOFIT regroupe deux communautés scientifiques, engagées respectivement dans la caractérisation et le fonctionnement des écosystèmes forestiers actuels ainsi que dans la reconstruction des changements passés de la végétation et du climat.

2 Époque géologique s’étendant sur les 10000 dernières années.

3 Le programme spatial Landsat a été créé en 1960 par l'Agence spatiale américaine afin d'observer la Terre.

4 Corine Land Cover est une base de données européenne d'occupation biophysique des sols.

5 L'élevage pastoral est un système extensif où les troupeaux pâturent sur de grandes étendues. Le système d'exploitation pastoral est basé sur la mobilité des troupeaux, qui se déplacent selon les disponibilités en pâturages - en liaison avec la distribution saisonnière de la pluie - et en points d'eau.

6 Ainsi sont désignés les premiers Peuls installés dans les hauts plateaux de l'Adamaoua au Cameroun. On les retrouve nombreux à cheval au Cameroun et au Nigeria.

7 C'est un sous-groupe de Peuls (ou Foulbé, dénomination utilisée au nord du Cameroun) appelé aussi M’bororo.

8 Unité territoriale à laquelle restent attachés les pasteurs lorsqu'ils se déplacent à l'occasion de la transhumance et vers laquelle ils reviennent.

9 Unité territoriale déterminée et reconnue vers laquelle convergent les pasteurs en saison sèche à la recherche des ressources pastorales.

10 Les Foulata et les M'bororo font aussi partie de l'ethnie des Peuls. Les premiers sont des éleveurs nomades ou semi-nomades, alors que les seconds pratiquent le nomadisme.

11 Une placette est une surface délimitée et localisée avec précision, dans laquelle des inventaires de végétation sont effectués plusieurs fois durant une période.

12 Un faciès écologique est un ensemble de caractères physionomiques particuliers au sein d'une formation végétale. Ces caractéristiques particulières, en général très localisées, permettent de distinguer des spécificités originales au sein des espèces végétales d'un même habitat.

\section{RÉFÉRENCES}

Achoundong, G., Youta Happi, J., Guillet, B., Bonvallot, J. et Kamgang Belaya, V. (2000). Formation et évolution des recrus en savane. Dans S. Servant-Vildary et M. Servant (dir.), Dynamique à long terme des écosystèmes forestiers intertropicaux (p. 115-119). Paris : UNESCO.

Andigué, J. (1999). Mise en place d'un système d'information géographique comme base d'une stratégie pour une meilleure gestion des espaces ruraux : cas du canton Bongor rural au sud-ouest du Tchad (Thèse de doctorat inédite). Université de Paris I, Paris, France.

Antrop, M. (2014). Interpreting diversity in the European landscape: A comment on perspective essays by Agnoletti and Schnitzler. Landscape and Urban Planning, 126, 81-83. doi: 10.1016/j.landurbplan.2014.02.013

Aoudou Doua, S. et Tchotsoua, M. (2010). Cartographie par télédétection de l'occupation du sol dans la haute vallée de la Bénoué. Annales de la FALSH de l'Université de N'Gaoundéré (Cameroun), 12, 101-112.

Boutrais, J. (1980). L'arbre et le bœuf en zone soudano-guinéenne, Cabiers de l'ORSTOM, série Sciences humaines, 17(3-4), 235-246. Repéré à www.documentation.ird.fr/hor/fdi:00995

Cabot, J. (1965). Le bassin du Moyen Logone. Paris: ORSTOM. Repéré à http://horizon.documentation.ird.fr/ exl-doc/pleins_textes/pleins_textes_6/Mem_cm/10420.pdf

Commission of the European Communities (CEC). (1993). Projet Corine Land Cover (document technique). $1^{\text {re }}$ partie. Bruxelles : CEC.

Djangrang, M. et Retaillé, D. (2015). L’extension récente des vergers dans la vallée de Kou au Tchad : enjeux socioenvironnementaux. Revue africaine d'anthropologie, 18, 19-31. Repéré à www.revues-ufhb-ci.org/ fichiers/FICHIR_ARTICLE_1959.pdf

Fredou, A. et Maley, J. (2000). Le contenu pollinique de l'atmosphère dans les forêts du sud Cameroun près de Yaoundé : résultats préliminaires. Dans M. Servant et S. Servant-Vildary (dir.), Dynamique à long terme des écosystèmes forestiers intertropicaux (p. 139-148). Paris : UNESCO. 
Lanly, J.P. (1982). Les ressources forestières tropicales - Étude FAO : Forêts 30. Organisations des nations unies pour l'alimentation et l'agriculture, Rome, Italie. Repéré à www.fao.org/3/ap450f/ap450f00.pdf

Marchal, J.-Y. (1980). Arbres et brousses du paysage soudano-sahélien : dynamique des formations végétales au nord de la Haute Volta. Cabiers de l'ORSTOM, série Sciences humaines, 17(3-1), 137-149. Repéré à www.documentation.ird.fr/hor/fdi:00986

Ntoupkap, J.-C. (1994). Étude de la dynamique d'une savane arborée dans la zone soudano-sabélienne (Nord-Cameroun) sous les effets combinés du pâturage, du feu et de la coupe de bois (Mémoire de maîtrise inédit). Université de Montpellier III, Montpellier, France.

Organisation des Nations unies pour l'alimentation et l'agriculture (FAO). (1994). Introduction à la gestion conservatoire de l'eau, de la biomasse et de la fertilité des sols (CGES). Bulletin pédologique de la FAO, 70, Rome : FAO. Repéré à http:// horizon.documentation.ird.fr/exl-doc/pleins_textes/divers11-03/41504.pdf

Organisation des Nations unies pour l'alimentation et l'agriculture (FAO). (1997). Africover : classification de l'occupation du sol. Rome : FAO.

Raimond, C., Robert, T. et Garine, E. (2012). Quels facteurs de l'évolution de l'agrobiodiversité au cours du XXe siècle dans le bassin du lac Tchad? Bilan du programme Plantadiv. Paris : France.

Réounodji, F. (2003). Espaces, sociétés rurales et pratiques de gestion des ressources naturelles dans les savanes du sud-ouest du Tchad : vers une intégration agriculture-élevage (Thèse de doctorat inédite). Université Paris 1 Panthéon-Sorbonne, Paris, France.

Schwartz, D., Dechamps R., Elenga H., Mariotti A. et Vincens A. (1996). Les savanes d'Afrique Centrale : des écosystèmes à l'origine complexe, spécifiques de l'Holocène supérieur. Dans Dynamique à long terme des écosystèmes forestiers intertropicaux : résumés (p. 179-182). Paris : ORSTOM. Repéré à http://horizon.documentation.ird.fr/ exl-doc/pleins_textes/pleins_textes_7/carton01/010007591.pdf

Seignobos, C. (1980). Des fortifications végétales dans la zone soudano-sahélienne (Tchad et Nord-Cameroun). Cabier de l'ORSTOM, série Sciences humaines, 17(3-4), 199-222. Repéré à horizon.documentation.ird.fr/exldoc/pleins_textes/pleins_textes_4/sci_hum/00992.pdf

Sougnabé, P. (2010). Pastoralisme en quête d'espaces en savane tchadienne : des Peuls autour de la forêt classée de Yamba Berté (Thèse de doctorat inédite). École des hautes études en sciences sociales de Paris, Paris, France.

Sougnabé, P., Ali Brahim, B. et Hassane Mahamat, H. (2004). Étude sur les pratiques pastorales dans et autour de la forêt classée de Yamba Berté. Farcha, Tchad : Laboratoire de recherches vétérinaires et zootechniques (LRVZ) de Farcha.

Tchotsoua, M. (1999). L'homme et la dynamique des paysages sur la dorsale de l'Adamaoua. Le Flamboyant, 50, 26-39.

Téblé, W. (2014). Péjorations climatiques et évolutions des galeries forestières actuelles dans les départements de Kouh (Logone oriental, Tchad), Annales XIII, Mélange 3, Compte 2, vol. 2.

Yao Sadaiou Sabas, B., Barbier, N., Bamba, I., Traore, D., Lejoly, J. et Bogaert, J. (2009). Dynamique paysagère en milieu de transition forêt-savane ivoirienne. Bois et forêts des tropiques, 299(1), 15-25. Repéré à www.researchgate.net/publication/323203516_Dynamique_paysagere_en_milieu_de_transition_foret-savane_ivoirienne

Youta Happi, J. (1998). Arbres contre graminées : la lente invasion de la savane par la forêt au centre-Cameroun. Paris : Université Paris-Sorbonne (Paris IV). 